\title{
Quantum state manipulation of single atom magnets using the hyperfine interaction
}

\author{
Patrick Robert Forrester $\odot,{ }^{1,2, *}$ François Patthey, ${ }^{1}$ Edgar Fernandes, ${ }^{1}$ Dante Phillipe Sblendorio $\odot,{ }^{1}$ \\ Harald Brune $\mathbb{0},^{1, \dagger}$ and Fabian Donat Natterer ${ }^{1}{ }^{1,2, \ddagger}$ \\ ${ }^{1}$ Institute of Physics, École Polytechnique Fédérale de Lausanne, Station 3, CH-1015 Lausanne, Switzerland \\ ${ }^{2}$ Department of Physics, University of Zurich, Winterthurerstrasse 190, CH-8057 Zurich, Switzerland
}

(Received 15 August 2019; published 19 November 2019)

\begin{abstract}
The magnetic quantum states of holmium single atom magnets on $\mathrm{MgO}(100)$ have proven extremely robust when exposed to high magnetic fields and temperatures up to $35 \mathrm{~K}$. Here we address the stability of Ho at small magnetic fields, where the hyperfine interaction creates several avoided level crossings. Using spin-polarized scanning tunneling microscopy, we demonstrate quantum state control via Landau-Zener tunneling and stable magnetization at zero field. Our observations indicate a total spin ground state of $J_{z}= \pm 8$. Combined quantum and classical control render Ho a promising qubit candidate.
\end{abstract}

DOI: 10.1103/PhysRevB.100.180405

The appeal of single atom magnets (SAMs) and single molecule magnets (SMMs) lies in their promising roles in magnetic data storage [1-5] and as qubits for quantum information processing [6,7]. As classical magnetic bits, SAMs and SMMs have demonstrated magnetic bistability $[1,3,4,8]$, even above liquid nitrogen temperatures [5], readability and writability [1,9-12], and self-assembly into periodic arrays $[4,8]$. For quantum information processing, the precise control over their local environment, intrinsic isolation, and relative ease of on-surface deposition render SAMs and SMMs attractive qubit candidates [13-20]. Early experiments have shown promise, so far culminating with an experimental realization of Grover's search algorithm on a single molecule [7]. Further realizations of SAM/SMM qubits would open up directly accessible Hilbert spaces whose dimensionalities exceed those of proof-of-principle systems.

Holmium SAMs are of particular interest for conventional and quantum information processing. Compared to SMMs, the combined thermal and magnetic field stability of $\mathrm{Ho} / \mathrm{MgO} / \mathrm{Ag}(100) \mathrm{SAMs}$ is unmatched. The magnetic relaxation time is $T_{1} \approx 1 \mathrm{~min}$ at $45 \mathrm{~K}$ in an out-of-plane field of $8 \mathrm{~T}$ [12]. When adsorbed on $\mathrm{MgO}$, holmium atoms have a $J=8$ ground state manifold [3], split by the crystal field of the on-top oxygen adsorption site [21]. Long-lived magnetization lifetimes $(\approx 1500 \mathrm{~s}$ at $10 \mathrm{~K}$ and $10 \mathrm{mT})$ were first measured with x-ray magnetic circular dichroism (XMCD), which also suggested that the largest contribution to the ground state wave function comes from the total spin $J_{z}= \pm 7$

\footnotetext{
${ }^{*}$ Present address: Department of Physics, Harvard University, Cambridge, Massachusetts 02138, USA.

${ }^{\dagger}$ Corresponding author: harald.brune@epfl.ch

${ }^{\ddagger}$ Corresponding author: fabian.natterer@uzh.ch
}

Published by the American Physical Society under the terms of the Creative Commons Attribution 4.0 International license. Further distribution of this work must maintain attribution to the author(s) and the published article's title, journal citation, and DOI. state [3]. Spin-polarized scanning tunneling microscopy (SPSTM) identified three voltage thresholds for magnetization switching, and electron spin resonance STM measured the total magnetic moment as $(10.1 \pm 0.1) \mu_{\mathrm{B}}[11,12]$. These observations are compatible with pure $J_{z}= \pm 7$ and \pm 8 ground states, which should become distinguishable at low magnetic fields [12]. However, relaxation times have been measured down to only $10 \mathrm{mT}$ by XMCD [3], and SP-STM measurements used large magnetic fields $(B \geqslant 200 \mathrm{mT})$ to polarize the paramagnetic tip apex. Therefore it has remained unknown whether this SAM is stable at zero field, and, related to this question, whether its magnetic ground state is $J_{z}= \pm 7$ or \pm 8 .

In this Rapid Communication, we demonstrate the zerofield stability of $\mathrm{Ho} / \mathrm{MgO}$ using antiferromagnetic SP-STM tips that enable robust spin contrast in the absence of an external magnetic field [22]. At small magnetic fields we observe a significant number of state reversals that we trace back to the nuclear spin of Ho. The hyperfine interaction creates avoided level crossings that couple the positive and negative total spin manifolds allowing quantum tunneling of the magnetization at specific nonzero magnetic field values $[23,24]$. For the crystal field of $\mathrm{Ho} / \mathrm{MgO}$, the avoided level crossings of the $J_{z}= \pm 8$ ground state explain all of our observations, while the absence of observable avoided level crossings for $J_{z}= \pm 7$ cannot account for switching at small nonzero fields. Through magnetic field sweep experiments, we show the capacity to invert or retain the atom's magnetic state with high fidelity via Landau-Zener tunneling [23]. This state control, the stability of both spin manifolds, and the coupling between the two manifolds at avoided level crossings highlight the potential of Ho SAMs as a platform for quantum information processing and conventional data storage.

We perform SP-STM measurements using a home-built low-temperature STM, operating below $p=1 \times 10^{-10} \mathrm{mbar}$ and at $T=4.7 \mathrm{~K}$ [25]. We grow 1.5 monolayers (ML) of $\mathrm{MgO}$ by exposing an atomically clean $\mathrm{Ag}(100)$ crystal at $773 \mathrm{~K}$ to a $\mathrm{Mg}$ flux from a Knudsen cell in an oxygen partial pressure of $1.33 \times 10^{-6} \mathrm{mbar}$ at a growth rate of $0.2 \mathrm{ML} / \mathrm{min}[3,26]$. We dose Ho atoms onto the sample at 


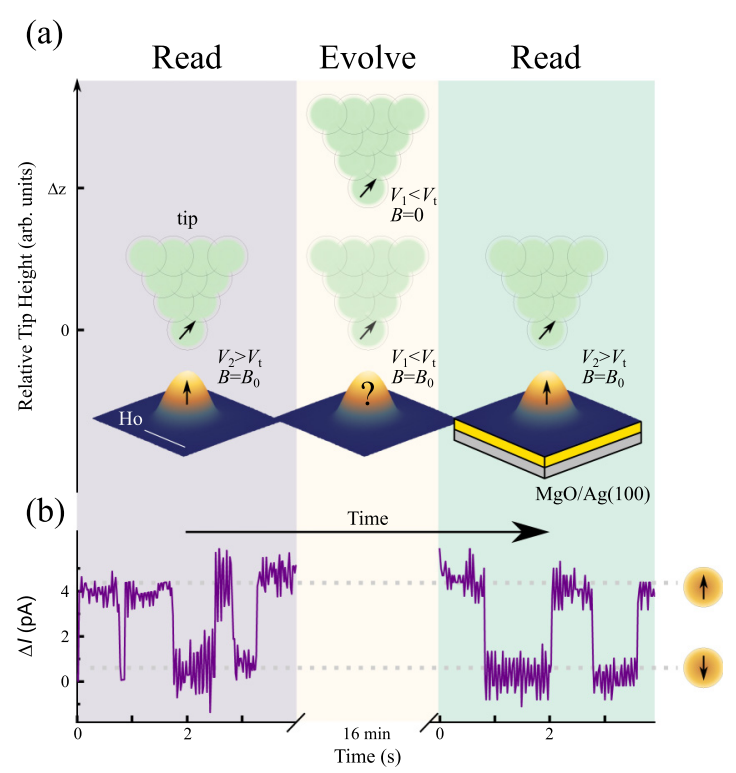

FIG. 1. (a) Schematic of SP-STM tip (green) centered above a Ho atom on an $\mathrm{MgO}$ thin film on $\mathrm{Ag}(100)$ (STM image recorded at $I_{t}=104 \mathrm{pA}, V=-130 \mathrm{mV}$, white scale bar $2 \mathrm{~nm}$ ). We read the holmium's magnetic state, Up or Down, before and after its evolution by switching it at a tunnel voltage above the switching threshold $V_{2}>$ $V_{t}=73 \mathrm{mV}$ (left and right panels). (b) Current-time traces showing two-state switching $\left(I_{\mathrm{set}}=100 \mathrm{pA}, V_{2}=-130 \mathrm{mV}, T=4.7 \mathrm{~K}\right.$, feedback open). When at tunnel distances, the antiferromagnetic tip exposes the atom to a stray field, $B_{0}$; when sufficiently retracted the field is zero. In the example shown, the Up state is preserved for 16 min at $V_{1}<V_{\mathrm{t}}$ and $B=B_{0}$.

$10 \mathrm{~K}$ using a thoroughly degassed $e$-beam evaporator. We use antiferromagnetic $\mathrm{Mn}_{88} \mathrm{Ni}_{12}$ tips [22]. Their out-of-plane spin polarization is tested by observing spin contrast on Ho atoms as they are switched by tunnel electrons above the threshold voltage $V_{t}=73 \mathrm{mV}[11,12,22]$. To adjust the magnetic field, the STM tip is retracted $5 \mathrm{~nm}$ for single retract/approach experiments and by $1 \mathrm{~nm}$ for multiple retractions/approaches. For all single retract/approach experiments, except those with a hold time of $60 \mathrm{~s}$, the tip is retracted in a single step, i.e., within a few microseconds. For the $60 \mathrm{~s}$ data and the multiple retract/approach cycles, the tip is retracted linearly $(d z / d t=$ const) in $100 \mathrm{~ms}$. For the state inversion measurements, the tip is retracted by $1.3 \mathrm{~nm}$ over the course of $20 \mathrm{~s}$ such that $d B / d t \approx$ const (assuming $B \propto z^{-3}$ ). Spin Hamiltonian calculations are performed using the EASYSPIN package [27] and the Steven's parameters of Natterer et al. [12]. We correct for unseen switching events from finite preamplifier bandwidth in Fig. 2(c), assuming a Markovian probability of state reversal and using measured residence times for the Up and Down states. Error bars are calculated using the Agresti-Coull method for binomial processes.

Figure 1 shows our SP-STM experimental setup. We read Ho's magnetic state by recording the telegraph signal in the tunnel current $I_{t}$ at voltages $V_{2}>V_{t}$ (left and right panels). We monitor its evolution at $V_{1}<V_{t}$, either exposing the atom to the tip stray field, $B_{0}$, or at zero magnetic field by retracting the tip very far (middle panel). As seen in Fig. 1(b) tunnel magnetoresistance gives rise to a contrast $\Delta I_{t}=4 \mathrm{pA}$ between the
Up and Down states at a tunnel current of $I_{t}=100 \mathrm{pA}$. Here, we leave the atom behind in the Up state, let it evolve for $\Delta t=$ $16 \mathrm{~min}$ at $V_{1}<V_{t}$ and $B=B_{0}$. The first switch during the subsequent read phase shows that the atom remained in the Up state, therefore that state was stable for $16 \mathrm{~min}$. This stability is notable, as the atom was exposed to smaller fields than in former STM studies [11,12]. During tunneling conditions, we believe the tip stray field is comparable to those measured for similar antiferromagnetic SP-STM tips $(\approx 100 \mathrm{mT})[28,29]$.

In order to investigate the stability of the magnetic quantum states down to zero external field, we retract the tip during the evolution phase by $\Delta z=5 \mathrm{~nm}$ [Fig. 2(a)]. We assume a field decay of $B \propto z^{-3}$ and expect a stray field below the Earth's magnetic field already at $\Delta z=1 \mathrm{~nm}$. Figure 2(b) shows the four possible outcomes for each individual read-evolve at zero field-read experiment, state conservation: Up $\rightarrow$ Up (green) or Down $\rightarrow$ Down (red), or state switching: Up $\rightarrow$ Down (blue) or Down $\rightarrow$ Up (orange). The zero-field stability of individual Ho atoms is evident from inspection of Fig. 2(c). For all hold times $\Delta t$, the Ho atoms retain their magnetic state in the overwhelming number of cases. Starting in the Up state, the state preservation averaged over all hold times $(\Delta t)$ is $99_{-2}^{+1} \%$, i.e., unity within the statistical error of the overall 884 experiments carried out with five different tips. Starting in the Down state yields a $\Delta t$-averaged state preservation of $(91 \pm 5) \%$ and thus few, albeit statistically significant, state reversals. For both initial states, the reversal rate does not increase with hold time, indicative of magnetic bistability.

To investigate whether the magnetic field sweeps caused by moving the tip back and forth from tunnel distance to full retraction induce state reversals, we retract and approach the tip $N$ times in the evolve phase before reading the state [dotted blue trace in Fig. 2(a)]. This is equivalent to $N$ magnetic field sweeps from $B=B_{0}$ to $0 \mathrm{~T}$ and back to $B_{0}$. Figure $2(\mathrm{~d})$ displays the results of 691 such experiments with four different microtips. It shows a clear increase in switching. Starting in the Down state, the abundance of state switches jumps from $3_{-3}^{+6} \%$ for $N=1$ to $(30 \pm 3) \%$ for $N=20$. Sweeping more does not further increase switching; we find $28_{+4}^{+5} \%$ for $N=100 \mathrm{retract} /$ approach cycles. Also for the Up state, the abundance of state switches becomes more noticeable when performing more field sweeps. It goes from $0_{-0}^{+3} \%$ at $N=1$, to ( $2 \pm 1) \%$ at $N=20$, then to $3_{-1}^{+2} \%$ at $N=100$. We conclude that the magnetic state reversals are caused by magnetic field sweeps.

The $N=1$ experiments show an asymmetry of spin reversal occurrences depending on the initial state. Multiple retract/approach cycles can be viewed as repeated binomial processes with inequivalent probabilities. For increasing $N$ values, we would first expect growth, then saturation of state reversal with different saturation values for the two starting states [Fig. 2(d)]. We believe the asymmetry for the $N=1$ cycle can be primarily explained by differences in the thermal occupation, though the origin of the asymmetry does not change the expected behavior after many retractions.

Our observations can be reconciled when considering the nuclear spin $I$ and its coupling to the total angular momentum of the electrons by the hyperfine interaction, $A$. Holmium has $I=7 / 2$ with $100 \%$ natural abundance. Similar values of $A$ have been reported for different Ho-containing single 
(a)

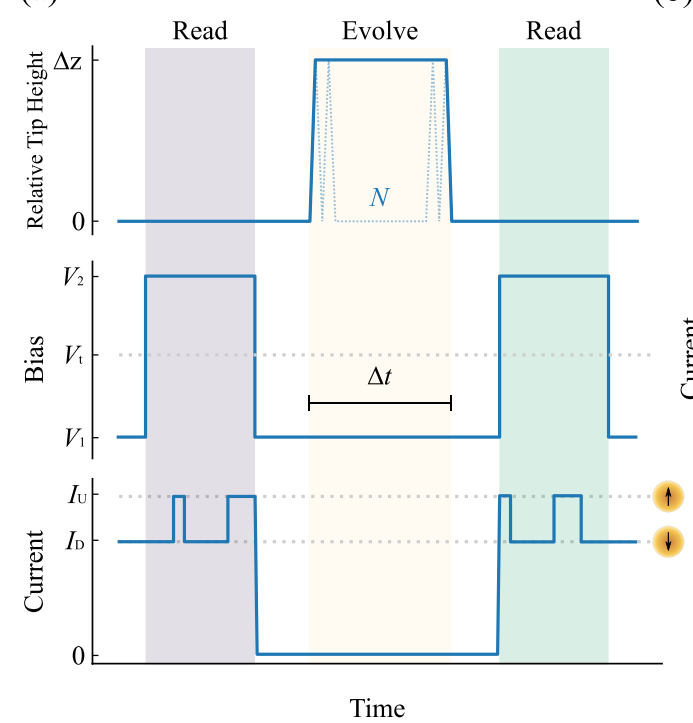

(c)

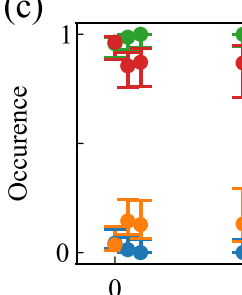

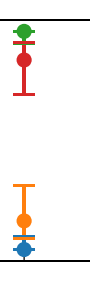

(b)
Time

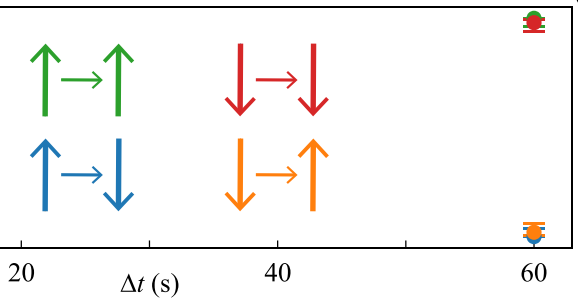

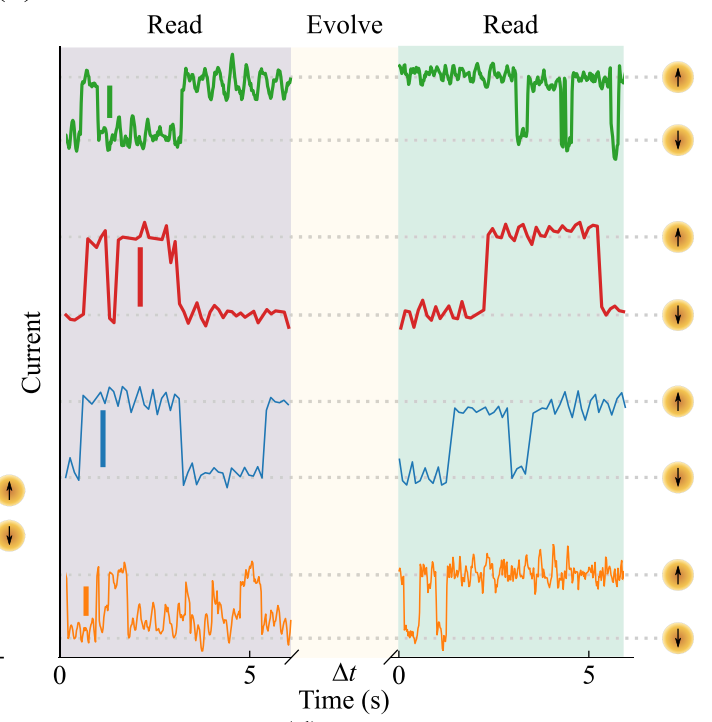

(d)

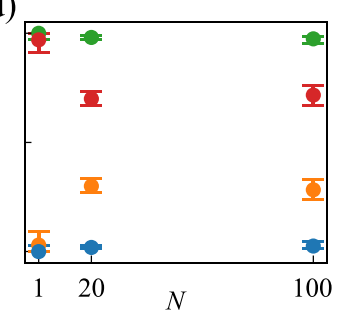

FIG. 2. (a) Schematic of tip height (upper), bias voltage (middle), and tunnel current (lower) as a function of time. The evolve phase lasts $\Delta t$, during which the tip is either held at $\Delta z$, giving $B=0$ (full curve), or it is retracted and approached $N$ times during $\Delta t$ in order to sweep the field $N$ times from $B_{0}$ to 0 and back to $B_{0}$ (dashed curve). (b) Current-time traces for state conservation, Up $\rightarrow$ Up (green) or Down $\rightarrow$ Down (red), or state switching, $\mathrm{Up} \rightarrow$ Down (blue) or Down $\rightarrow \mathrm{Up}$ (orange) $\left(I_{\text {set }}=100 \mathrm{pA}\right.$, upper two traces $V=-120 \mathrm{mV}$, lower two traces $V=130 \mathrm{mV}$, feedback open, vertical scale bars $5 \mathrm{pA}$ ). (c) Abundance of state conservation and switching for $N=1$ as a function of $\Delta t$. (d) Abundance of state conservation and switching as a function of $N$.

ion magnets $(3.3 \mu \mathrm{eV}$ [30], $3.4 \mu \mathrm{eV}$ [31,32], and $3.7 \mu \mathrm{eV}$ [33]). We therefore use $A=3.7 \mu \mathrm{eV}$ for $\mathrm{Ho} / \mathrm{MgO}$ in our spin Hamiltonian calculations [27]. We use the crystal field parameters previously determined by field-dependent SP-STM measurements [12]. Figures 3(a) and 3(b) show the extended Zeeman diagrams of the lowest energy total spin states for the two plausible ground states of $J_{z}= \pm 8$ and \pm 7 . For the first model, the hyperfine interaction leads to eight avoided level crossings at nonzero magnetic fields, marked as red circles in Fig. 3(a). They exhibit a tunnel splitting of $\Delta=0.1 \mu \mathrm{eV}$, as seen in the enlarged crossing in Fig. 3(e). Figure 3(c) displays the lowest energy zero-field crossing. Without the hyperfine interaction $(A=0)$ we find the expected zero-field mixing and an avoided level crossing (dashed red curves), while the hyperfine interaction creates a real crossing and thus zero-field stability (full orange curves). This is the case for all $J_{z}= \pm 8$ zero-field crossings. For the $J_{z}= \pm 7$ model, the zero-field crossings are real, irrespective of the hyperfine interaction [see Fig. 3(d) for the lowest energy crossing]. Therefore in both models, albeit for different reasons, none of the zero-field crossings are mixed, implying zero-field stability.

To identify the magnetic ground state, we examine avoided level crossings at nonzero field values. While the $J_{z}= \pm 8$ model exhibits eight such crossings, the $J_{z}= \pm 7$ model does not host avoided level crossings within the resolution of our calculations. Sweeping the magnetic field through an avoided level crossing results in a nonzero probability of magnetic state reversal [24]. Therefore, the increased occurrence of state reversals with an increased number of magnetic field sweeps (tip retract/approach cycles) leads us to conclude that the Ho ground state must be $J_{z}= \pm 8$.

Note, the magnitude of $A$ does not influence which crossings are avoided and only minimally scales the tunnel splitting. Our qualitative conclusions thus remain valid for different values of $A$. Also note, some Ho SMMs exhibit $J_{z}= \pm 7$ electronic ground states in fourfold symmetric crystal fields and host sizable avoided level crossings but with significantly larger transverse crystal field coefficients $\left(B_{4}^{4}\right.$ and $\left.B_{4}^{6}\right)[2,30]$. We show in Fig. 3(f) a magnified crossing for the $J_{z}= \pm 7$ model where an avoided level crossing would occur were either transverse crystal field parameter, $B_{4}^{4}$ or $B_{4}^{6}$, of comparable magnitude to those of previously observed molecular systems.

To demonstrate Landau-Zener tunneling at avoided level crossings [23], we repeat the experiment described in Fig. 2(a) with different magnetic field sweep rates for approaching and retracting. We set the fast magnetic sweep rate 

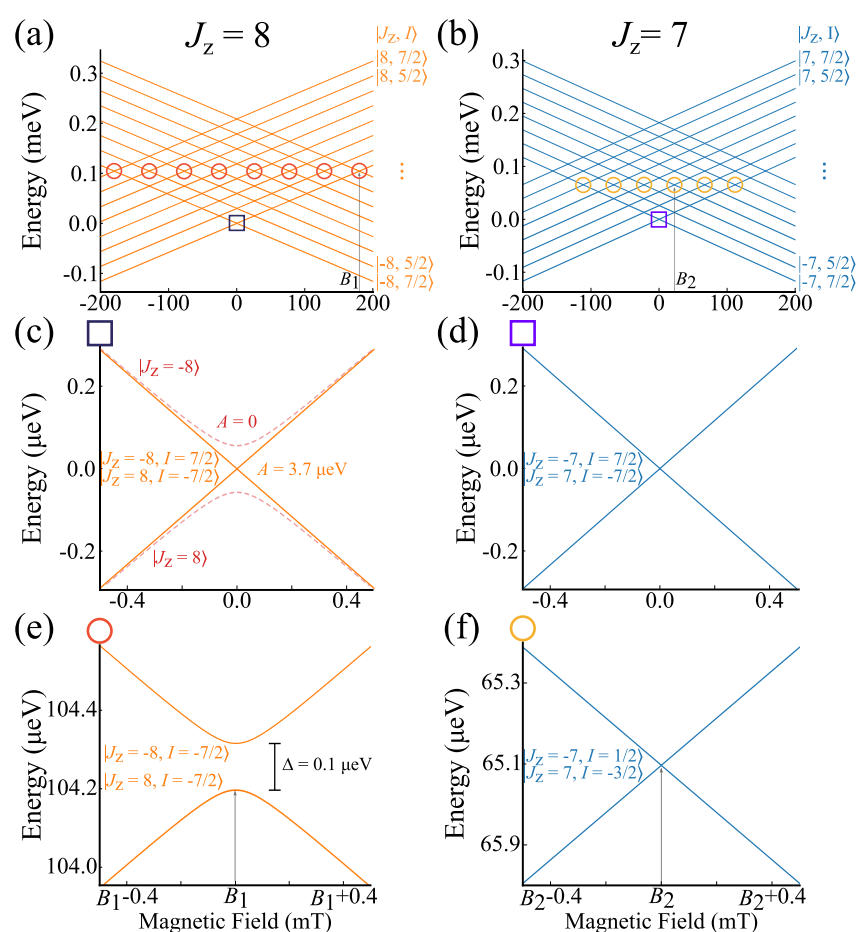

(d)
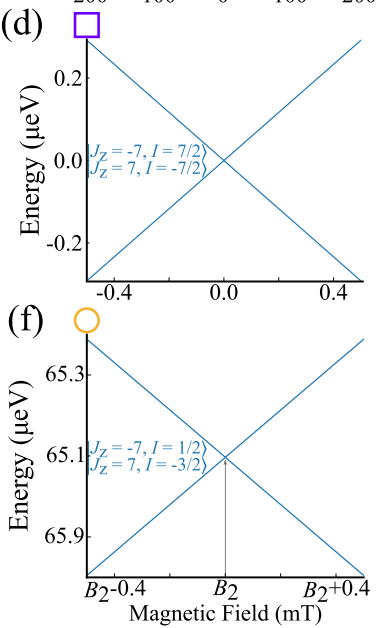

FIG. 3. (a) Extended Zeeman diagram for the $J_{z}= \pm 8$ ground state highlighting the eight avoided level crossings (red circles) and the lowest energy zero-field crossing (dark-purple square). (b) Same as (a) for $J_{z}= \pm 7$ model with lowest energy zero-field crossing (light-purple square) and locations where avoided level crossings were found for molecular Ho systems (gold circles). (c) Lowest energy zero-field crossing with (solid orange) and without (dashed red) nuclear spin for the $J_{z}= \pm 8$ model. (d) Real level crossing at zero field for the $J_{z}= \pm 7$ model. (e) Representative avoided level crossing for the $J_{z}= \pm 8$ model. (f) Our crystal field yields a real level crossing for the $J_{z}= \pm 7$ model, where Ho single ion magnets had avoided level crossings.

to $d B / d t=10^{5}-10^{7} \mathrm{~T} / \mathrm{s}$ and the slow one to $d B / d t=$ $10^{-4} \mathrm{~T} / \mathrm{s}$. The probability of magnetic state reversal is $p=1-\exp \left[-\pi^{2} \Delta^{2} /\left(h g_{\mathrm{eff}} J_{z} d B / d t\right)\right]$ [23], where $g_{\mathrm{eff}}$ is the Landé $g$ factor, which we chose to match the measured magnetic moment $\left(g_{\text {eff }} J_{z}=10.1 \mu_{\mathrm{B}}\right)[11]$ and $h$ is the Planck constant. The probability of switching is $100 \%$ for the slow sweep and only several percent for the fast one. Performing a fast and slow sweep consecutively (or vice versa) thus results in a net reversal of the spin state (adiabatic rapid passage [34]). Table I shows the observed magnetic state reversal for these protocols. Both show state reversal occurrences on the order of 50\% regardless of the initial state, in accordance with the
TABLE I. Switching probabilities for state inversion through Landau-Zener tunneling. A total of 110 experiments have been performed with the same tip.

\begin{tabular}{lccllc}
\hline \hline & \multicolumn{2}{c}{ Slow-fast } & & \multicolumn{2}{c}{ Fast-slow } \\
\cline { 2 - 3 } \cline { 6 - 6 } & Expt. & Theory & & Expt. & Theory \\
\hline $\mathrm{Up} \rightarrow$ Down & $55_{-16}^{+15} \%$ & $57 \%$ & & $35_{-15}^{+19} \%$ & $43 \%$ \\
Down $\rightarrow \mathrm{Up}$ & $48_{-17}^{+17} \%$ & $43 \%$ & & $59_{-23}^{+20} \%$ & $57 \%$ \\
\hline \hline
\end{tabular}

Landau-Zener tunneling process. Note, we expect occurrences near $50 \%$ (instead of 100\%) as a maximum of four out of the eight nuclear spin levels for each manifold host avoided level crossings for the field values used in this experiment ( $B=B_{0} \rightarrow B=0 \rightarrow B=B_{0}$, where $\left.B_{0}>0\right)$. We observe different occurrences of magnetic state reversal depending on the initial Ho state, in agreement with the thermal occupation probability of the states that traverse avoided level crossings for the two spin manifolds (57\% and 43\%, respectively). Accordingly, the efficiency of magnetic state reversal for each initial Ho state reverses when the sweep order is reversed. The initial Ho state is randomized with tunnel electrons whose energies are orders of magnitude larger than the spacing between hyperfine states, justifying thermal distribution arguments. Expanding the sweep range to $B_{0}$ to $-B_{0}$ would facilitate high-fidelity state inversion. Note that this is a tunnel electron-free scheme to control the magnetic state of SAMs and operates solely via the action of a magnetic field on the Ho level structure.

Our experimental study and numerical modeling show the zero-field stability of Ho SAMs and the existence of avoided level crossings at certain nonzero magnetic field values, allowing us to identify a ground state of $J_{z}= \pm 8$. The avoided level crossings couple the positive and negative spin manifolds, allowing transitions via Landau-Zener tunneling. We show how Landau-Zener tunneling could be used for state inversion without requiring tunneling electrons. Control of LandauZener tunneling, the stability of both spin manifolds, a large nuclear quadrupole moment, and a microwave accessible 64dimension Hilbert space make Ho SAMs an interesting qubit candidate.

P.R.F. thanks Michael Kopreski and Adrianne Zhong for useful discussions. We thank Stefano Rusponi, Nicholas Chilton, Mario Ruben, and Daniel Loss for fruitful discussions. P.R.F. acknowledges support from the Fulbright US Student Program. We further acknowledge funding from the Swiss National Science Foundation under Projects No. PP00P2_176866 (F.D.N.) and No. 200020_176932 (D.P.S.).
[1] R. Sessoli, D. Gatteschi, A. Caneschi, and M. A. Novak, Nature (London) 365, 141 (1993).

[2] N. Ishikawa, M. Sugita, and W. Wernsdorfer, J. Am. Chem. Soc. 127, 3650 (2005).

[3] F. Donati, S. Rusponi, S. Stepanow, C. Wäckerlin, A. Singha, L. Persichetti, R. Baltic, K. Diller, F. Patthey, E.
Fernandes, J. Dreiser, Z. Sljivancanin, K. Kummer, C. Nistor, P. Gambardella, and H. Brune, Science 352, 318 (2016).

[4] R. Baltic, M. Pivetta, F. Donati, C. Wäckerlin, A. Singha, J. Dreiser, S. Rusponi, and H. Brune, Nano Lett. 16, 7610 (2016).

[5] F. Guo, B. M. Day, Y. Chen, M. Tong, A. Mansikkamäki, and R. A. Layfield, Science 362, 1400 (2018). 
[6] M. N. Leuenberger and D. Loss, Nature (London) 410, 789 (2001).

[7] C. Godfrin, A. Ferhat, R. Ballou, S. Klyatskaya, M. Ruben, W. Wernsdorfer, and F. Balestro, Phys. Rev. Lett. 119, 187702 (2017).

[8] C. Wäckerlin, F. Donati, A. Singha, R. Baltic, S. Rusponi, K. Diller, F. Patthey, M. Pivetta, Y. Lan, S. Klyatskaya, M. Ruben, H. Brune, and J. Dreiser, Adv. Mater. 28, 5195 (2016).

[9] R. Vincent, S. Klyatskaya, M. Ruben, W. Wernsdorfer, and F. Balestro, Nature (London) 488, 357 (2012).

[10] F. Troiani, C. Godfrin, S. Thiele, F. Balestro, W. Wernsdorfer, S. Klyatskaya, M. Ruben, and M. Affronte, Phys. Rev. Lett. 118, 257701 (2017).

[11] F. D. Natterer, K. Yang, W. Paul, P. Willke, T. Choi, T. Greber, A. J. Heinrich, and C. P. Lutz, Nature (London) 543, 226 (2017).

[12] F. D. Natterer, F. Donati, F. Patthey, and H. Brune, Phys. Rev. Lett. 121, 027201 (2018).

[13] C. J. Wedge, G. A. Timco, E. T. Spielberg, R. E. George, F. Tuna, S. Rigby, E. J. L. McInnes, R. E. P. Winpenny, S. J. Blundell, and A. Ardavan, Phys. Rev. Lett. 108, 107204 (2012).

[14] K. Bader, D. Dengler, S. Lenz, B. Endeward, S. D. Jiang, P. Neugebauer, and J. v. Slageren, Nat. Commun. 5, 5304 (2014).

[15] M. Atzori, L. Tesi, E. Morra, M. Chiesa, L. Sorace, and R. Sessoli, J. Am. Chem. Soc. 138, 2154 (2016).

[16] K. Yang, Y. Bae, W. Paul, F. D. Natterer, P. Willke, J. L. Lado, A. Ferrón, T. Choi, J. Fernández-Rossier, A. J. Heinrich, and C. P. Lutz, Phys. Rev. Lett. 119, 227206 (2017).

[17] G. Czap, P. J. Wagner, F. Xue, L. Gu, J. Li, J. Yao, R. Wu, and W. Ho, Science 364, 670 (2019).

[18] E. Moreno-Pineda, S. Klyatskaya, P. Du, M. Damjanovic, G. Taran, W. Wernsdorfer, and M. Ruben, Inorg. Chem. 57, 9873 (2018).
[19] W. Wernsdorfer and M. Ruben, Adv. Mater. 31, 1806687 (2019).

[20] B. Verlhac, N. Bachellier, L. Garnier, M. Ormaza, P. Abufager, R. Robles, M. L. Bocquet, M. Ternes, N. Lorente, and L. Limot, Science 366, 6465 (2019).

[21] E. Fernandes, F. Donati, F. Patthey, S. Stavric, Z. Sljivancanin, and H. Brune, Phys. Rev. B 96, 045419 (2017).

[22] P. R. Forrester, T. Bilgeri, F. Patthey, H. Brune, and F. D. Natterer, Rev. Sci. Instrum. 89, 123706 (2018).

[23] C. Zener, Proc. R. Soc. London, Ser. A. 137, 696 (1932).

[24] D. Gatteschi and R. Sessoli, Angew. Chem., Int. Ed. 42, 268 (2003).

[25] R. Gaisch, J. K. Gimzewski, B. Reihl, R. R. Schlittler, M. Tschudy, and W. D. Schneider, Ultramicroscopy 42-44, 1621 (1992).

[26] J. Pal, M. Smerieri, E. Celasco, L. Savio, L. Vattuone, and M. Rocca, Phys. Rev. Lett. 112, 126102 (2014).

[27] S. Stoll and A. Schweiger, J. Magn. Reson. 178, 42 (2006).

[28] A. A. Khajetoorians, B. Baxevanis, C. Hübner, T. Schlenk, S. Krause, T. O. Wehling, S. Lounis, A. Lichtenstein, D. Pfannkuche, J. Wiebe, and R. Wiesendanger, Science 339, 55 (2013).

[29] F. D. Natterer, F. Patthey, T. Bilgeri, P. R. Forrester, N. Weiss, and H. Brune, Rev. Sci. Instrum. 90, 013706 (2019).

[30] R. Giraud, W. Wernsdorfer, A. M. Tkachuk, D. Mailly, and B. Barbara, Phys. Rev. Lett. 87, 057203 (2001).

[31] S. Ghosh, S. Datta, L. Friend, S. Cardona-Serra, A. Gaita-Ariño, E. Coronado, and S. Hill, Dalton Trans. 41, 13697 (2012).

[32] M. Shiddiq, D. Komijani, Y. Duan, A. Gaita-Arino, E. Coronado, and S. Hill, Nature (London) 531, 348 (2016).

[33] Y. C. Chen, J. L. Liu, W. Wernsdorfer, D. Liu, L. F. Chibotaru, X. M. Chen, and M. L. Tong, Angew. Chem., Int. Ed. 56, 4996 (2017).

[34] E. Treacy, Phys. Lett. 27A, 421 (1968). 